Chirurg 2016 $87: 442$

DOI 10.1007/s00104-016-0190-5

Online publiziert: 13. April 2016

(c) Springer-Verlag Berlin Heidelberg 2016

CrossMark

F. Rauchfuß - U. Settmacher

Klinik für Allgemein-, Viszeral- und Gefäßchirurgie, Universitätsklinikum Jena, Jena, Deutschland

\title{
Rezidive der primären sklerosierenden Cholangitis nach Lebertransplantation
}

transplantation bei PSC“ identifiziert, wobei schlussendlich 565 Patienten in die Auswertung einbezogen werden konnten. Das mittlere Follow-up betrug 9 Jahre.

347 dieser Patienten (61,4\%) hatten eine chronisch-entzündliche Darmerkrankung, wobei die Fälle mit Colitis ulcerosa die absolute Mehrzahl ( $\mathrm{n}=$ 306; 88,2\%) ausmachten. Die Diagnose wurde bereits bei 235 Patienten vor der Transplantation gestellt, wobei bei 35 Individuen eine Kolektomie vor der Transplantation erfolgt, bei 4 während der Lebertransplantation und bei 37 post transplantationem.

Bei 81 Patienten musste die Diagnose PSC-Rezidiv gestellt werden. Eine univariate Analyse stellte das Vorhandensein einer Colitis ulcerosa als größten Prädiktor für das Auftreten eines PSC-Rezidivs, unabhängig vom Zeitpunkt der Diagnosestellung, heraus.

Daneben waren die angewandte Immunsuppression (Cyclosporin-basiert), der Zeitraum der Lebertransplantation (1990-2000 vs. 2001-2010), das Empfängeralter und der Zeitpunkt einer etwaigen Kolektomie Risikofaktoren für das Auftreten eines PSC-Rezidivs. Eine multivariate Analyse erbrachte das Vorhandensein einer Colitis ulcerosa post transplantationem sowie ein junges Patientenalter als PSC-Rezidiv-Risikofaktoren.

Das Auftreten eines Rezidivs der PSC war mit einem deutlich verschlechterten Transplantat- und Patientenüberleben vergesellschaftet.
Diskussion. Die vorliegende Studie betrachtet das bislang größte publizierte Kollektiv an PSC-Patienten mit der Fragestellung des Rezidivs der Grunderkrankung. Insbesondere das Vorhandensein einer Colitis ulcerosa nach der Lebertransplantation, sei es de novo oder vorbestehend, ist ein Risikofaktor für PSCRezidive. Der zugrunde liegende Pathomechanismus kann aufgrund des retrospektiven Studiendesigns nicht abschließend geklärt werden.

Fazit. Die Assoziation zwischen PSC und chronisch-entzündlichen Darmerkrankungen ist bekannt, unklar und umstritten ist nach wie vor der ideale Zeitpunkt bzw. die Notwendigkeit einer Kolektomie bei Transplantationspatienten mit PSC und einer begleitenden Colitis ulcerosa. In der vorliegenden Studie konnte ein klarer Zusammenhang zwischen einer Colitis und dem Auftreten eines PSC-Rezidivs herausgearbeitet werden.

\section{Korrespondenzadresse}

\section{PD Dr. F. Rauchfuß}

Klinik für Allgemein-, Viszeral- und Gefäßchirurgie, Universitätsklinikum Jena Erlanger Allee 101, 07747 Jena, Deutschland falk.rauchfuss@med.uni-jena.de

Interessenkonflikt. F. Rauchfuß und U. Settmacher geben an, dass kein Interessenkonflikt besteht. 